\title{
Cuidados paliativos nas mídias sociais: revisão integrativa de literatura
} Palliative care in social media: integrative literature review

\section{Atención paliativa en medios sociales: revisión integrativa de la literatura}

Priscila Kelly da Silva Neto ${ }^{1, a}$

priscila.baldonado@gmail.com | https://orcid.org/o0oo-0002-0553-2714

Jacqueline Resende Boaventura ${ }^{1, b}$

jacque_boaventura@yahoo.com.br | https://orcid.org/o0oo-0002-0466-4459

Juliana Dias Reis Pessalacia ${ }^{1, c}$

juliana@pessalacia.com | https://orcid.org/oooo-0003-0468-2283

Maristela Rodrigues Marinho ${ }^{1, d}$

mmaristelammarinho@gmail.com | https://orcid.org/o0oo-0003-4610-6911

Fernando Ribeiro dos Santos $^{1, e}$

fernanndoribeiro@hotmail.com | https://orcid.org/o0oo-0002-8913-5205

\footnotetext{
${ }^{1}$ Universidade Federal de Mato Grosso do Sul, Campus de Três Lagoas. Três Lagoas, MS, Brasil.

a Especialização em Auditoria e Gestão em Serviço de Saúde pela Instituição de Ensino Capacitação e Pós-Graduação.

${ }^{\mathrm{b}}$ Mestrado em Enfermagem pela Universidade Federal de Mato Grosso do Sul.

c Doutorado em Enfermagem pela Universidade de São Paulo.

d Especialização em Gestão em Saúde pela Universidade Federal de São Paulo.

e Graduando em Medicina pela Universidade Federal de Mato Grosso do Sul.
}

\section{RESUMO}

Este artigo traz o estudo realizado com o objetivo de identificar as publicações sobre como os cuidados paliativos são abordados nas mídias sociais. Trata-se de uma revisão integrativa de literatura, com pesquisa de artigos nas bases Medical Literature Analysis and Retrieval System Online (Medline), Literatura LatinoAmericana e do Caribe em Ciências da Saúde (Lilacs) e Scientific Eletronic Library Online (SciELO), em português, inglês e espanhol. Foram selecionados quinze artigos que atendiam aos critérios de seleção propostos, e são aqui apresentados por meio da construção de um quadro sinóptico, contendo título do estudo, periódico, ano de publicação e abordagem dos cuidados paliativos nas mídias sociais. Os resultados dos estudos demonstram que os cuidados paliativos necessitam ser melhor difundidos nas mídias sociais. Entretanto, tal evolução deve ocorrer com cautela, de modo a evitar riscos de comprometer a qualidade das informações e a respeitar questões éticas que envolvem pacientes e profissionais de saúde. Cabe destacar o papel do enfermeiro em ações de educação em saúde relacionadas com cuidados paliativos.

Palavras-chave: Cuidados paliativos; Mídias sociais; Internet; Assistência à saúde; Enfermagem. 


\section{ABSTRACT}

The study presented in this article aims to identify publications which reveal how palliative care is approached on social media. It is an integrative literature review, using search of articles in the bases Medical Literature Analysis and Retrieval System Online (Medline), Latin American and Caribbean Health Sciences Literature (Lilacs) and Scientific Electronic Library Online (SciELO), in Portuguese, English and Spanish languages. Fifteen articles that met the proposed selection criteria were selected, and they are presented here through the construction of a synoptic table, containing the title of the study, the journal in which it was published, its publication date and the specific approaches to palliative care in social media. The results of the studies demonstrate that the palliative care needs to be better disseminated on social media. However, caution should be taken while such dissemination occuring, in order to prevent risks damaging the quality of information and to follow ethical issues involving patients and health professionals. The role of nurses in health education actions regarding the palliative care should be highlighted.

Keywords: Palliative care; Social media; Internet; Health care; Nursing.

\section{RESUMEN}

El estudio presentado en este artículo tiene el objetivo de identificar publicaciones sobre cómo se aborda el cuidado paliativo en las redes sociales. Es una revisión literaria integrativa, haciendo una búsqueda de artículos en las bases del Medical Literature Analysis and Retrieval System Online (Medline), Literatura Latinoamericana y del Caribe en Ciencias de la Salud (Lilacs) y Scientific Eletronic Library Online (SciELO), en portugués, inglés y español. Se seleccionaron quince artículos que cumplían los criterios de selección propuestos, y ellos son presentados aquí a través de la construcción de un cuadro sinóptico, que contiene el título del estudio, la revista en la cual ha sido publicado, el año de publicación y el abordaje de los cuidados paliativos en las redes sociales. Los resultados de los estudios demuestran que los cuidados paliativos deben ser mejor difundidos en las redes sociales. Sin embargo, dicha difusión debe ocurrir con precaución para evitar riesgos de perjudicar la calidad de la información y para cumplir las cuestiones éticas que involucran pacientes y profesionales de la salud. El papel de los enfermeros y de las enfermeras en las acciones de educación en salud relacionadas con cuidados paliativos debe ser destacado.

Palabras clave: Cuidados paliativos; Medios sociales; Internet; Atención de la salud; Enfermería.

Contribuição dos autores:

Concepção e desenho do estudo: Priscila Kelly da Silva Neto; Jacqueline Resende Boaventura; Juliana Dias Reis Pessalacia; Fernando Ribeiro dos Santos.

Aquisição, análise ou interpretação dos dados: Priscila Kelly da Silva Neto; Jacqueline Resende Boaventura; Juliana Dias Reis Pessalacia.

Redação do manuscrito: Priscila Kelly da Silva Neto; Jacqueline Resende Boaventura; Juliana Dias Reis Pessalacia; Maristela Rodrigues Marinho; Fernando Ribeiro dos Santos.

Revisão crítica do conteúdo intelectual: Priscila Kelly da Silva Neto; Jacqueline Resende Boaventura; Juliana Dias Reis Pessalacia; Maristela Rodrigues Marinho; Fernando Ribeiro dos Santos.

Declaração de conflito de interesses: não há.

Fontes de financiamento: não houve.

Considerações éticas: não há.

Agradecimentos/Contribuições adicionais: não há.

Histórico do artigo: submetido: 09 dez. 2019 | aceito: 30 jun. 2020 | publicado: 17 dez. 2020.

Apresentação anterior: não houve.

Licença CC BY-NC atribuição não comercial. Com essa licença é permitido acessar, baixar (download), copiar, imprimir, compartilhar, reutilizar e distribuir os artigos, desde que para uso não comercial e com a citação da fonte, conferindo os devidos créditos de autoria e menção à Reciis. Nesses casos, nenhuma permissão é necessária por parte dos autores ou dos editores. 


\section{INTRODUÇÃO}

O avanço dos cuidados paliativos (CP) em todo o mundo trouxe para a esfera pública intensos debates relacionados aos estigmas negativos relacionados aos mesmos. Frequentemente, associa-se CP com abandono, morte e desesperança, sendo necessárias estratégias educacionais visando quebrar tabus e conscientizar a população sobre esta modalidade de cuidados. Em 2011, uma pesquisa de opinião pública de 800 adultos revelou que 70\% desconhecem os CP. Assim, embora tais cuidados estejam mais acessíveis em diversos países, a maioria das pessoas ainda desconhece seus objetivos e efeitos na saúde das populações.

$\mathrm{O}$ conceito de $\mathrm{CP}$ foi recentemente reformulado pela International Association for Hospice and Paliattive Care (IAHPC), sendo considerados como cuidados holísticos ativos voltados para pacientes em sofrimento intenso relacionado à saúde, em especial àqueles no final da vida. O objetivo da prática dos $\mathrm{CP}$ é, portanto, melhorar a qualidade de vida dos pacientes, de seus familiares e de seus cuidadores, podendo inclusive coexistir com o tratamento da doença em $\mathrm{si}^{2}$.

Em contrapartida, o avanço tecnológico das últimas décadas tem permitido um maior acesso mundial à internet e, consequentemente, aos sites de mídias sociais (MS). Trata-se de plataformas online, geralmente móveis, que visam à troca de informações e experiências por seus usuários. As MS mais conhecidas atualmente são o Facebook, o Twitter, o Youtube, o Instagram e o Linkedin, além de fóruns online voltados para comunidades específicas (por exemplo, Patientslikeme, Mumsnet). Neste contexto, vem aumentando cada dia mais a quantidade de MS voltadas para a área de saúde e bem-estar, podendo ser consideradas preocupações sociais dominantes ${ }^{3}$.

As informações sobre saúde têm sido ativamente pesquisadas, compartilhadas, comunicadas e discutidas por meio das MS. Sabe-se que esse tipo de troca de informações online pode beneficiar os usuários em aspectos de acesso imediato a informações de interesse em saúde, suporte emocional, psicológico e tomada de decisão relacionada à saúde .

Os CP se inserem neste contexto buscando nas MS um espaço coletivo para discussão pública e quebra de paradigmas quanto a esta modalidade de cuidados, visto que, a temática da morte e do morrer é historicamente tida como tema 'tabu' para discussão pública. Assim, observa-se nos últimos anos que, cada vez mais, pacientes terminais, suas famílias e profissionais de CP e de saúde têm falado abertamente em MS sobre o assunto ${ }^{3}$.

Desde o surgimento das MS, publicações de autores, conselhos e associações alertam sobre os perigos do uso deste recurso para profissionais de saúde e pacientes ${ }^{5}$. Entretanto, sabe-se que recentemente os conteúdos sobre $\mathrm{CP}$ têm se apresentado como de qualidade pelas $\mathrm{MS}^{1}$.

Contudo, a partir de buscas nas bases disponíveis na Biblioteca Virtual em Saúde (BVS), não foram identificadas publicações recentes de revisões sistemáticas ou integrativas que relacionem a temática dos $\mathrm{CP}$ e as MS. Deste modo, tornam-se relevantes estudos que busquem sumarizar este assunto, buscando-se conhecer as potencialidades e fragilidades que envolvem o compartilhamento de informações e experiências sobre CP em tais mídias. Assim, este estudo teve como objetivo identificar as publicações nacionais e internacionais sobre como os CP são abordados nas MS.

\section{MÉTODOS}

Trata-se de uma revisão integrativa de literatura (RIL), de modo a responder à seguinte questão: quais são os estudos que abordam os CP no contexto das MS? Para tal, foram percorridas as seguintes etapas: estabelecimento da hipótese e objetivos da RIL, estabelecimento de critérios de inclusão e exclusão de artigos (seleção da amostra), definição das informações a serem extraídas dos artigos selecionados, análise dos resultados, discussão e apresentação dos resultados e apresentação da revisão ${ }^{6}$. 
A busca foi realizada no mês de março de 2019, sendo consultadas as bases de dados Medical Literature Analysis and Retrieval System Online (Medline), Literatura Latino- americana e do Caribe em Ciências da Saúde (Lilacs) e a Scientific Eletronic Library Online (SciELO). Foram utilizados os descritores disponíveis Decs/Mesh: 'Cuidados Paliativos' OR 'Palliative care', 'Mídias sociais' OR ‘Social media', combinados por meio do operador booleano AND. As estratégias de busca utilizadas para a seleção dos artigos foram: no PubMed - 'Palliative care’ AND 'Social Media’; na Lilacs e SciELO - 'Cuidados Paliativos' AND 'Mídias Sociais'.

Foram incluídos artigos de periódicos, com texto completo disponível, em língua portuguesa, inglesa e espanhola, que contemplavam a questão norteadora de estudo, publicados no período de 2014 a 2019. Optou-se pelos últimos seis anos, após identificar-se, através de pesquisas prévias às bases consultadas, que este período contemplava um maior número de publicações na temática. Foram excluídos artigos duplicados nas bases e que não apresentavam resumos. Desenvolveu-se um formulário a fim de facilitar a análise e a caracterização dos artigos da amostra. Apresentaram-se, no formulário utilizado, as seguintes informações: identificação do artigo e autores; fonte de localização; objetivos; delineamento e síntese dos resultados.

A seleção foi feita por meio da leitura criteriosa do título e do resumo online. Posteriormente, os estudos selecionados foram lidos na íntegra e avaliados se respondiam ou não à questão norteadora da pesquisa.

Inicialmente foram encontrados 77 artigos, sendo: o na base de dados Lilacs, o na biblioteca SciELO e 77 na Medline. Após a leitura dos títulos e resumos de todos os 77 artigos, foram excluídos: 17 estudos que se encontravam fora do período de busca; 35 artigos por não atenderem à temática proposta; três estudos publicados em outros idiomas; dois editoriais, um resumo, um na forma de comentário, outro que não se encontrava com metodologia claramente descrita, e outro que não estava com texto disponível na íntegra, totalizando 61 artigos excluídos. Os 16 artigos restantes foram, posteriormente, lidos na íntegra, excluindose mais um artigo pelo fato de não responder à questão norteadora. Dessa forma, a amostra constituiu-se em 15 artigos (100\%) que atendiam aos critérios de seleção propostos.

Os resultados foram apresentados por meio da construção de um quadro sinóptico, contendo título do estudo, periódico, ano de publicação e abordagem dos CP nas MS. Quanto à síntese das publicações encontradas nas bases de dados analisadas, foi possível agrupá-las, distribuindo-as em três categorias de análise temática. Posteriormente, os principais resultados foram relacionados com dados presentes na literatura sobre a temática. 
Observam-se, abaixo, os resultados encontrados a partir da aplicação dos mecanismos de busca:

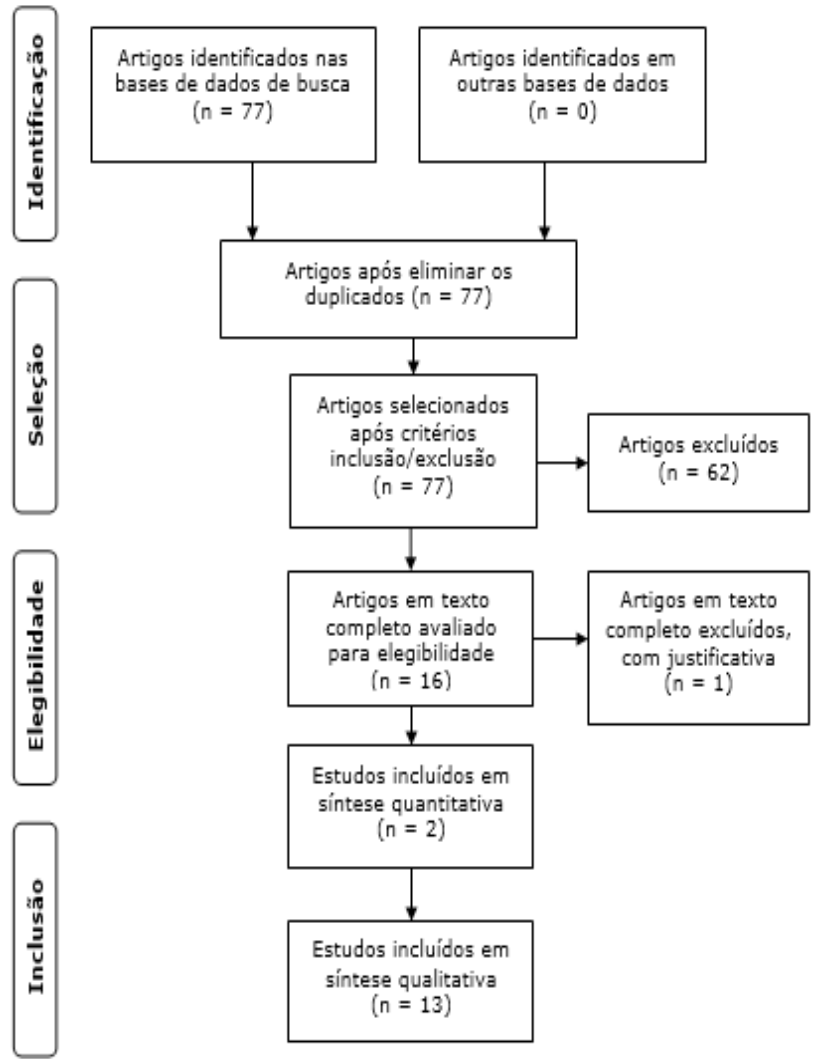

Figura 1 - Fluxograma dos mecanismos de busca Fonte: Elaborado pelos autores (2019).

\section{RESULTADOS E DISCUSSÃO}

Em relação à caracterização dos artigos analisados, foram publicados nos anos de 2014 e 2019, sendo dois (13,3\%) publicados em 2019, três (20\%) em 2018, quatro (26,6\%) em 2017, três (20\%) em 2016, dois (13,3\%) em 2015 e um (6,6\%) em 2014. No que concerne ao conteúdo do periódico, 12 (80\%) foram publicados em revistas voltadas a CP; dois (13,3\%), em revistas voltadas para publicações interdisciplinares; e um (6,6\%), em revista científica de amplo aspecto (Tabela 1).

A fim de facilitar a visualização dos resultados encontrados, foi realizada a construção de um quadro sinóptico (Quadro 1). Por meio dele, poderão ser visualizados os títulos dos artigos encontrados, os títulos dos periódicos, ano de publicação e a abordagem dos CP nas MS em cada uma das pesquisas. 
Tabela 1 - Características dos artigos incluídos na revisão integrativa sobre abordagem dos cuidados paliativos em mídias sociais

\begin{tabular}{lcc} 
Características & No & \% \\
\hline Ano de Publicação & & \\
\hline 2019 & 2 & 13,3 \\
2018 & 3 & 20 \\
2017 & 4 & 26,6 \\
2016 & 3 & 20 \\
2015 & 2 & 13,3 \\
2014 & 1 & 6,6 \\
Conteúdo do Periódico & & \\
Revistas voltada para CP & 12 & 80 \\
Publicações interdisciplinares & 2 & 13,3 \\
\hline
\end{tabular}

Fonte: Elaborado pelos autores (2019).

Quadro 1 - Artigos incluídos na revisão integrativa sobre abordagem dos cuidados paliativos em mídias sociais

(continua)

\begin{tabular}{|l|l|l|l|}
\hline Código & \multicolumn{1}{|c|}{ Título } & $\begin{array}{c}\text { Periódico } \\
\text { e Ano de } \\
\text { Publicação }\end{array}$ & \multicolumn{1}{|c|}{ Abordagem dos CP nas MS } \\
\hline A1 & $\begin{array}{l}\text { Social representation } \\
\text { of palliative care in the } \\
\text { Spanish printed media: a } \\
\text { qualitative analysis }\end{array}$ & $\begin{array}{l}\text { PLoS One, } \\
2019\end{array}$ & $\begin{array}{l}\text { Estudo com objetivo de analisar como os CP são } \\
\text { retratados nos jornais espanhóis, bem como a } \\
\text { contribuição da imprensa para sua representação social. } \\
\text { Aponta que as MS representam uma nova oportunidade } \\
\text { de envolvimento e diálogo contínuo com grupos públicos } \\
\text { e profissionais na temática dos CP. }\end{array}$ \\
\hline A2 & $\begin{array}{l}\text { The photographs of } \\
\text { meaning program for } \\
\text { pediatric palliative } \\
\text { caregivers: feasibility of } \\
\text { a novel meaning-making } \\
\text { intervention }\end{array}$ & $\begin{array}{l}\text { Am J Hosp } \\
\text { Palliat Care, } \\
2019\end{array}$ & $\begin{array}{l}\text { Foi realizado um estudo com o objetivo de avaliar a } \\
\text { viabilidade e o engajamento de cuidadores de pacientes } \\
\text { em CP pediátricos, através do Photographs of Meaning } \\
\text { Program. Durante um período de nove semanas, os } \\
\text { participantes criaram e compartilharam narrativas } \\
\text { fotográficas através das mídias sociais. Os resultados } \\
\text { sugerem que o programa proposto de utilização de MS } \\
\text { foi uma intervenção prática com potencial de melhoria } \\
\text { de vida por meio de cuidados paliativos pediátricos. }\end{array}$ \\
\hline A3 & $\begin{array}{l}\text { \#Deathbedlive: the end-of- } \\
\text { life trajectory, reflected in a } \\
\text { cancer patient's tweets }\end{array}$ & $\begin{array}{l}\text { BMC Palliat } \\
\text { Care, } 2018\end{array}$ & $\begin{array}{l}\text { Estudo com objetivo de examinar as narrativas digitais } \\
\text { de pacientes terminais, publicadas nas MS, e revela o } \\
\text { potencial dos dados de MS e da bioetnografia digital } \\
\text { para iluminar as experiências vividas, as estratégias } \\
\text { de enfrentamento e as necessidades de suporte aos } \\
\text { pacientes terminais, sugerindo novas oportunidades } \\
\text { para melhorar os cuidados paliativos personalizados por } \\
\text { intermédio de mais pesquisas. }\end{array}$ \\
\hline
\end{tabular}


(continua)

\begin{tabular}{|c|c|c|c|}
\hline Código & Título & $\begin{array}{l}\text { Periódico } \\
\text { e Ano de } \\
\text { Publicação }\end{array}$ & Abordagem dos CP nas MS \\
\hline A4 & $\begin{array}{l}\text { Evaluating palliative care } \\
\text { resources available to the } \\
\text { public using the internet } \\
\text { and social media }{ }^{1}\end{array}$ & $\begin{array}{l}\text { Am J Hosp } \\
\text { Palliat Care, } \\
2018\end{array}$ & $\begin{array}{l}\text { Estudo com objetivo de identificar recursos na internet } \\
\text { e MS sobre CP e avaliar as informações transmitidas. } \\
\text { Os principais mecanismos de busca da internet, como } \\
\text { o Google e o YouTube, fornecem informações valiosas } \\
\text { sobre as informações que o público recebe sobre os CP. } \\
\text { O desenvolvimento futuro de recursos da internet para } \\
\text { CP deve considerar a inclusão de crianças e enfatizar } \\
\text { os CP para todas as doenças que limitam a vida e não } \\
\text { somente para o câncer. }\end{array}$ \\
\hline A5 & $\begin{array}{l}\text { Pain and policy studies } \\
\text { group: two decades } \\
\text { of working to address } \\
\text { regulatory barriers to } \\
\text { improve opioid availability } \\
\text { and accessibility around the } \\
\text { world9 }^{9}\end{array}$ & $\begin{array}{l}\text { J Pain } \\
\text { Symptom } \\
\text { Manage, } 2018\end{array}$ & $\begin{array}{l}\text { O estudo apresenta o Pain \& Policy Studies Group (PPSG), } \\
\text { um programa de pesquisa global da Universidade de } \\
\text { Wisconsin Carbone Cancer Center, o qual tem trabalhado } \\
\text { para cumprir sua missão de melhorar o alívio da dor } \\
\text { mediante o acesso equilibrado a opiáceos em todo } \\
\text { o mundo. O referido programa estabeleceu, entre } \\
\text { as ferramentas, o uso de de redes sociais, incluindo } \\
\text { Facebook, Twitter, YouTube, Pinterest, LinkedIn e } \\
\text { blogs, com o objetivo de aumentar a visibilidade de } \\
\text { suas atividades globais. Avançou seu uso e escopo } \\
\text { de ferramentas de redes sociais e planeja continuar a } \\
\text { divulgação das ações usando essas ferramentas. }\end{array}$ \\
\hline A6 & $\begin{array}{l}\text { An exploratory investigation } \\
\text { of hospice marketing: How } \\
\text { are palliative care providers } \\
\text { marketing their services? }\end{array}$ & $\begin{array}{l}\text { Health Mark Q, } \\
2017\end{array}$ & $\begin{array}{l}\text { Estudo com objetivo de examinar as iniciativas } \\
\text { estratégicas de marketing que as organizações de } \\
\text { CP têm utilizado. Muitas empresas utilizam de mídia } \\
\text { eletrônica e social, tais como, Facebook, Blogs e Twitter } \\
\text { como estratégia de marketing em CP. Estas ferramentas } \\
\text { são frequentes para pacientes em fim de vida com idade } \\
\text { geralmente superior a } 75 \text { anos. No entanto, podem ser } \\
\text { apropriadas para outras populações, como médicos, } \\
\text { familiares e até mesmo sociedade e políticos. }\end{array}$ \\
\hline A7 & $\begin{array}{l}\text { Can digital stories go where } \\
\text { palliative care research has } \\
\text { never gone before? } \\
\text { A descriptive qualitative } \\
\text { study exploring the } \\
\text { application of an emerging } \\
\text { public health research } \\
\text { method in an indigenous } \\
\text { palliative care context }{ }^{11}\end{array}$ & $\begin{array}{l}\text { BMC Palliat } \\
\text { Care, } 2017\end{array}$ & $\begin{array}{l}\text { O estudo utiliza método de contar histórias através de } \\
\text { vídeos curtos em primeira pessoa, útil para explorar as } \\
\text { questões de CP. Este estudo tem o objetivo de explorar } \\
\text { a opinião de pessoas indígenas da tribo Maori, sobre } \\
\text { o cuidado no final da vida e, apesar de encontrar } \\
\text { barreiras, essa pesquisa defende o envolvimento } \\
\text { participativo da comunidade de pesquisa a fim de incluir } \\
\text { grupos indígenas em pesquisas sobre CP, e as MS } \\
\text { constituem uma importante ferramenta neste contexto. }\end{array}$ \\
\hline A8 & $\begin{array}{l}\text { Evaluating public } \\
\text { engagement for a } \\
\text { consensus development } \\
\text { conference }^{12}\end{array}$ & $\begin{array}{l}\text { J Palliat Med, } \\
2017\end{array}$ & $\begin{array}{l}\text { Com objetivo de incentivar e aumentar o engajamento } \\
\text { público, o Covenant Health Palliative Institute, em } \\
\text { parceria com as principais organizações de saúde do } \\
\text { Canadá, empreendeu uma iniciativa, conhecida como } \\
\text { Assuntos de cuidados paliativos (PCM - Palliative Care } \\
\text { Matters) para examinar as necessidades de CP em } \\
\text { todo o país. E para isso usou o Facebook e o Twitter de } \\
\text { modo a promover, compartilhar notícias e atualizações } \\
\text { da conferência, além de apresentar links, vídeos e fotos } \\
\text { relacionados à iniciativa PCM, e mostrou-se eficaz em } \\
\text { alcançar e envolver um público online. }\end{array}$ \\
\hline
\end{tabular}


(conclusão)

\begin{tabular}{|c|c|c|c|}
\hline Código & Título & $\begin{array}{l}\text { Periódico } \\
\text { e Ano de } \\
\text { Publicação }\end{array}$ & Abordagem dos CP nas MS \\
\hline A9 & $\begin{array}{l}\text { Use of podcast technology } \\
\text { to facilitate education, } \\
\text { communication and } \\
\text { dissemination in palliative } \\
\text { care: the development of } \\
\text { the AmiPal podcast }{ }^{13}\end{array}$ & $\begin{array}{l}\text { BMJ Support } \\
\text { Palliat Care, } \\
2017 .\end{array}$ & $\begin{array}{l}\text { Com objetivo de descrever o desenvolvimento de um } \\
\text { podcast de } \mathrm{CP} \text {, de acordo com as recomendações } \\
\text { internacionais para a qualidade de qualquer podcast e } \\
\text { para conduzir uma análise da escuta do podcast durante } \\
\text { um período de } 14 \text { meses. Um método que facilita a } \\
\text { discussão desses cuidados com o público global. O } \\
\text { trabalho futuro deve se concentrar no desenvolvimento } \\
\text { de conteúdo, métricas de qualidade e análise de impacto, } \\
\text { pois é provável que essa forma de comunicação digital } \\
\text { aumente e engaje a sociedade mais ampla. }\end{array}$ \\
\hline A10 & $\begin{array}{l}\text { Keep in touch (KIT): } \\
\text { perspectives on } \\
\text { introducing internet- } \\
\text { based communication and } \\
\text { information technologies in } \\
\text { palliative care }{ }^{14}\end{array}$ & $\begin{array}{l}\text { BMC Palliat } \\
\text { Care, } 2016\end{array}$ & $\begin{array}{l}\text { Este estudo teve como objetivo explorar a aceitabilidade } \\
\text { da introdução de tecnologias de comunicação e } \\
\text { informação baseadas na internet para pacientes } \\
\text { internados em uma unidade destinada a cuidados } \\
\text { paliativos. O uso de MS com a finalidade de pacientes } \\
\text { internados em unidades de CP manterem contato com } \\
\text { familiares e amigos é importante, pois a comunicação e } \\
\text { as informações poderiam beneficiar a sua qualidade de } \\
\text { vida e os cuidados recebidos. }\end{array}$ \\
\hline A11 & $\begin{array}{l}\text { Maintaining the social } \\
\text { flow of evidence-informed } \\
\text { palliative care: use and } \\
\text { misuse of YouTube }{ }^{15}\end{array}$ & $\begin{array}{l}\text { Indian J Palliat } \\
\text { Care, } 2016\end{array}$ & $\begin{array}{l}\text { O objetivo desse artigo foi destacar o papel do YouTube } \\
\text { no que se refere à prática, educação, pesquisa e } \\
\text { administração dos CP, considerando como recurso } \\
\text { de evidência pacientes e cuidadores, estudantes e } \\
\text { profissionais, provedores e formuladores de políticas } \\
\text { nos países em desenvolvimento. Destaca a importância } \\
\text { de se desenvolver políticas voltadas para CP e de } \\
\text { implementá-las com base em evidências por meio da } \\
\text { tecnologia de informação e redes sociais. }\end{array}$ \\
\hline A12 & $\begin{array}{l}\text { Palliative care research } \\
\text { centre's move into social } \\
\text { media: constructing a } \\
\text { framework for ethical } \\
\text { research, a consensus } \\
\text { paper }^{16}\end{array}$ & $\begin{array}{l}\text { BMJ Support } \\
\text { Palliat Care, } \\
2016\end{array}$ & $\begin{array}{l}\text { O artigo tem como objetivo observar as diretrizes } \\
\text { éticas enfrentadas pelos pesquisadores, ao abordarem } \\
\text { assuntos como as MS. Seu uso em pesquisas voltadas } \\
\text { para CP cria maiores desafios, que exigem diversas } \\
\text { considerações. Várias discussões culminaram em um } \\
\text { conjunto de diretrizes tomadas no sentido de garantir } \\
\text { que a pesquisa de mídias sociais seja conduzida de } \\
\text { maneira ajustada e eticamente aceitável. }\end{array}$ \\
\hline A13 & $\begin{array}{l}\text { Social media and palliative } \\
\text { medicine: a retrospective } \\
\text { 2-year analysis of global } \\
\text { Twitter data to evaluate } \\
\text { the use of technology to } \\
\text { communicate about issues } \\
\text { at the end of life }{ }^{17}\end{array}$ & $\begin{array}{l}\text { BMJ Support } \\
\text { Palliat Care, } \\
2015\end{array}$ & $\begin{array}{l}\text { O estudo tem por objetivo determinar a frequência com } \\
\text { que os sentimentos são expostos no Twitter, contendo } \\
\text { identificadores (hashtags) e/ou frases relacionadas aos } \\
\text { cuidados paliativos enviados pelos usuários durante } \\
\text { um período de dois anos. Observaram-se muitas } \\
\text { discussões sobre cuidados paliativos no Twitter, em sua } \\
\text { maioria positivas, apresentando uma nova oportunidade } \\
\text { para engajamentos e diálogos com grupos públicos e } \\
\text { profissionais. }\end{array}$ \\
\hline A14 & $\begin{array}{l}\text { Using social media in } \\
\text { supportive and palliative } \\
\text { care research }^{18}\end{array}$ & $\begin{array}{l}\text { BMJ Support } \\
\text { Palliat Care, } \\
2015\end{array}$ & $\begin{array}{l}\text { Este artigo tem por objetivo fornecer um resumo } \\
\text { abrangente das MS, incluindo seus fundamentos } \\
\text { teóricos, e exemplos recentes de usos bem-sucedidos } \\
\text { relacionados à pesquisa em saúde. Também descreve } \\
\text { as oportunidades e desafios em utilizar as MS nos } \\
\text { cuidados de fim da vida e os passos práticos que um } \\
\text { pesquisador pode seguir para recrutar pacientes usando } \\
\text { mídias sociais. Destaca o cuidado centrado no paciente } \\
\text { utilizando como uma das ferramentas as MS. }\end{array}$ \\
\hline
\end{tabular}

Fonte: Elaborado pelos autores (2019). 
Em relação à abordagem dos CP nas MS presentes nos artigos, notou-se que sete (46,6\%) discutiram a divulgação de ações e informações sobre os CP nas MS, cinco (33,3\%) abordaram as experiências pessoais divulgadas nas MS, três (20\%) trabalharam o uso das MS para a realização de pesquisas.

A partir das buscas nas bases de dados, emergiram três categorias temáticas, que representam como os CP são abordados nas MS, conforme os objetivos propostos, sendo elas: Categoria I - Divulgação de ações e informações em CP (A1, A4, A5, A6, A8, A10, A11), Categoria II - Experiências pessoais divulgadas em redes sociais (A3, A13, A2, A12, A15); Categoria III - Uso de MS para a pesquisa sobre CP (A7, A9, A14).

Identificou-se um maior número de estudos que abordavam os $\mathrm{CP}$ no contexto da divulgação de ações e informações, conforme descrito na categoria I.

Categoria I - Divulgação de ações e informações em CP: Nesta categoria, nota-se que entre os artigos examinados, a divulgação de ações e informações sobre os CP nas MS, tanto para profissionais de saúde quanto para a sociedade em geral, ainda é muito sutil e inconsistente. Uma das publicações identificadas ressalta a carência destas informações, apontando que na imprensa espanhola, por exemplo, elas têm uma presença substancial, porém ligadas a discursos políticos, estando as discussões ainda distantes da realidade da saúde; e que, ao invés de contribuirem para o entendimento, essas informações passam a ser uma barreira, uma vez que refletem diferentes interesses públicos?

Portanto, o assunto não tem sido abordado com o objetivo de divulgar informações médicas ou científicas, mas sim atrelado a movimentos sociais e políticos. Tais movimentos são necessários e se fazem constantemente presentes nas MS, confirmando-se que o assunto dos CP ainda se encontra atrelado à ausência de políticas públicas que garantam acesso a estes cuidados. Assim, os movimentos sociais se constituem como importantes alavancas para a mudança social, sendo a internet uma plataforma privilegiada para a construção social da autonomia. As MS se tornam ferramentas decisivas para organizar, mobilizar, deliberar, coordenar e decidir ${ }^{19}$.

Dentre os artigos que retratam esta categoria, é possível observar que opiniões públicas fizeram parte das MS, apresentando relatos que evidenciam a falta de conhecimento do público em geral quantos aos conceitos de CP. Conforme estudo realizado em 2014 com estagiários europeus de oncologia ginecológica, constatou-se que os profissionais de saúde envolvidos devem ser cautelosos quanto aos benefícios e danos que as MS podem causar, observando-se que a maioria dos entrevistados percebem a importância dos canais online para disseminação de informações, fóruns educativos e discussões clínicas e para o aumento do elo entre o profissional e a comunidade ${ }^{20}$.

Identificou-se também em uma das publicações desta revisão que $77 \%$ das citações nas MS remetem a tratamentos oncológicos e quase não citam crianças ou a utilização de ferramentas como o Twitter para facilitar a comunicação e alcançar países menos desenvolvidos com limites de acesso à internet, facilitando a divulgação dos CP e o uso de opióides ${ }^{1,9}$. Assim, observa-se que ainda se encontra restrita, nas MS, a divulgação de informações sobre outras patologias associadas aos CP. Os CP tradicionalmente são ofertados a pacientes oncológicos, devido principalmente aos inúmeros sintomas provocados por tal doença; entretanto, tais cuidados também deveriam ser ofertados às diversas doenças crônicas não transmissíveis, e também, não menos importante, a algumas doenças crônicas transmissíveis, tais como a Síndrome da imunodeficiência adquirida (aids) e casos de tuberculose resistentes ${ }^{21}$.

Além disso, deve-se ampliar o uso de ferramentas para a divulgação destas informações, visando atingir às populações menos desenvolvidas. Observa-se a pouca utilização do Twitter, a qual pode limitar o acesso a informações sobre os CP, pois o mesmo se diferencia de outras MS pelo fato de a maioria de suas mensagens serem públicas, curtas e abertas para o exame de qualquer pessoa conectada, diferentemente do Facebook, por exemplo, no qual o conteúdo é mais restrito a públicos específicos ${ }^{22}$.

Cabe mencionar ainda que, pelo fato de as MS ainda apresentarem um número restrito de informações sobre $\mathrm{CP}$, deve-se utilizá-las com muita cautela. Um estudo randomizado realizado na Alemanha junto a 
520 participantes identificou que os participantes tendiam a selecionar informações que sustentassem suas atitudes e crenças, o que não está de acordo com as evidências científicas atuais, indicando que os mesmos se utilizam de informações da web para atitudes persuasivas ${ }^{23}$. Portanto, o uso de MS em CP também acarreta riscos potenciais, incluindo a qualidade da informação, danos à imagem profissional, violação da confidencialidade do paciente e violação dos limites da relação médico-paciente ${ }^{20}$.

Categoria II - Experiências pessoais divulgadas em redes sociais: Para esta categoria emergiram cinco artigos identificados nas bases, os quais retratam as experiências pessoais divulgadas nas MS. Notou-se que os avanços tecnológicos da última década permitiram o acesso generalizado à internet na maioria dos países, com o uso cada vez mais comum de sites de $\mathrm{MS}^{13}$. Identifica-se mudança no modo como os profissionais de saúde interagem com educadores, colegas e o mundo exterior, utilizando as MS no desenvolvimento de comunicação interpessoal ${ }^{15}$.

Tal mudança no modo de interação se deve ao desenvolvimento da comunicação eletrônica e dos sistemas de informacionais, os quais propiciam uma crescente dissociação entre a proximidade espacial e o desempenho das funções. Acredita-se que no futuro, as cidades terão seus perfis alterados devido a este desenvolvimento ou poderão até mesmo desaparecer, sendo inevitável a emergência do teletrabalho enquanto esperança para se prevenir os megacongestionamentos nas cidades ${ }^{24}$.

Nesse contexto, torna-se necessário que os profissionais de saúde se envolvam com as novas formas digitais de conteúdo, incluindo MS online, tal como apontou um dos estudos identificados nesta revisão, compreendendo que embora muitas vezes não sejam consideradas baseadas em evidências são significativas para os pacientes, sendo chamadas de evidências experienciais ${ }^{15}$.

Nos artigos pesquisados nesta revisão, observou-se uma maior ênfase da abordagem da temática sobre o câncer. Historicamente, a morte e o morrer têm sido vistos como um tema tabu para discussão pública. No entanto, a disposição de falar abertamente sobre isso online por pacientes terminais, suas famílias e profissionais de CP e de saúde aumentou nos últimos anos, dando origem a um campo emergente de pesquisa ${ }^{5}$.

Outro estudo aponta a questão da morte e o morrer expressos em MS e destaca a importância do preparo dos profissionais da saúde frente às consequências inerentes ao mundo virtual. A existência de diários online é uma realidade, e após a morte serão mantidos para a posteridade; neles se observam relatos de enfrentamento de pacientes em terminalidade.

Vale destacar que uma nova função disponibilizada pelo Facebook permite que bisnetos visitem as páginas preenchidas por bisavós com sentimentos e mensagens expressas décadas anteriores. Essas ferramentas são conhecidas como 'serviços de memorialização online'. Assim, a internet tem proporcionado mudanças nos padrões de relacionamento em nossa sociedade, tornando-se necessárias discussões e reflexões críticas sobre os limites, benefícios e danos atrelados ao desenvolvimento das MS para a sociedade ${ }^{5}$.

Categoria III - Uso de MS para a pesquisa sobre CP: Esta categoria discorre sobre o uso das MS para a realização de pesquisas no âmbito dos CP. A análise dos artigos demonstrou experiências bem-sucedidas de pesquisadores ao utilizarem as MS como recurso. Os artigos abordam guias práticos para pesquisas em MS, uso de podcasts e a importância dos relatos em MS para se desenvolver abordagens de atuação junto a minorias sociais.

Contudo, vale destacar que as MS ainda se apresentam como um desafio para os diferentes métodos de pesquisa, despertando maior interesse de pesquisadores de várias áreas do conhecimento em investigar e compreender as interações mediadas por tecnologias ${ }^{25}$.

Um estudo de revisão bibliométrica, com o objetivo de descobrir a mudança temática e a evolução da utilização de MS para o campo da pesquisa em saúde, ressaltou que o uso de MS na pesquisa em saúde tem atraído cada vez mais a atenção das comunidades científicas. O estudo revelou como principais temas de pesquisa a análise do YouTube sobre eventos sexuais. As fontes de publicações mais prolíficas são o Journal 
of Medical Research, PLoS One, e Cyberpsychology, Behavior and Social Networking. Os Estados Unidos apresentaram um maior número de publicações utilizando as $\mathrm{MS}^{4}$.

Os resultados de um estudo demonstram que as MS podem ser usadas estrategicamente para aumentar a disseminação de artigos de pesquisa e para ter um feedback da população com vistas a propor soluções para problemas ${ }^{26}$.

Além disso, as MS também podem ser um meio para atrair participantes para os centros de pesquisa sobre $\mathrm{CP}$, visto que se considera o potencial da web para se impulsionar uma maior participação popular ${ }^{18}$.

\section{CONCLUSÃO}

A partir das discussões das categorias emergentes, apontam-se como potencialidades acerca da divulgação de informações relacionadas aos CP nas MS que tais tecnologias podem fortalecer a ligação entre profissional de saúde, pacientes e familiares. Além disso, o compartilhamento de experiências nas MS pode proporcionar evidências experienciais, colaborando para a realização de pesquisas científicas, e pode servir como recurso para a preservação de memórias familiares de falecidos. Outro possível benefício diz respeito ao alcance dessas informações por profissionais e pacientes de países menos desenvolvidos, os quais não dispõem de profissionais qualificados em CP.

Como fragilidades, as categorias comprovam que ainda são insuficientes as informações obtidas sobre $\mathrm{CP}$ nas MS e que, na maioria das vezes, as que são compartilhadas se encontram atreladas a discursos políticos ou à oncologia. Assim, mostra-se a necessidade de ampliação de tais informações para outras condições que merecem CP, não somente para a área de oncologia.

Tais informações também podem ser utilizadas indevidamente por pacientes que desejam reforçar atitudes e crenças sobre a doença de um modo a não se considerar evidências científicas, mas sim percepções de outros pacientes, por exemplo. Deste modo, ressalta-se que o aumento de informações sobre a temática deve ocorrer, considerando-se medidas de prevenção quanto aos riscos relacionados à qualidade da informação divulgada, danos à imagem profissional, violação da confidencialidade do paciente e violação dos limites da relação médico-paciente.

Deve-se considerar também a necessidade de capacitação profissional para o uso de tais tecnologias visando à divulgação de informações. As limitações deste estudo incluem a necessidade de abranger estudos com diferentes metodologias, devido à insuficiência de pesquisas que apresentem evidências científicas acerca das contribuições do uso das MS em CP.

\section{REFERÊNCIAS}

1. Claudio CH, Dizon ZB, October TW. Evaluating Palliative Care Resources Available to the Public Using the Internet and Social Media. Am J Hosp Palliat Care [Internet]. 2018 Set [cited 2019 Mar 5];35(9):117480. Available from: https://www.ncbi.nlm.nih.gov/pubmed/?term=Evaluating+ Palliative+Care+Resource s+Available+to+the+Public+Using+the+Internet+and+Social+Media.

2. IAHPC. Global Consensus based palliative care definition [Internet]. Houston, TX: The International Association for Hospice and Palliative Care. 2018 [cited 2020 Aug 10]. Available from https:// hospicecare.com/what-we-do/projects/consensus-based-definition-of-palliative-care/definition/.

3. Taylor J, Pagliari C. \#Deathbedlive: the end-of-life trajectory, reflected in a cancer patient's tweets. BMC Palliat Care [Internet]. 2018 Jan [cited 2019 Mar 5];17(1):17. Available from: https://www.ncbi.nlm.nih. gov/pubmed/?term $=\% 23$ Deathbedlive $\% 3 \mathrm{~A}+$ the+end-of-life+trajectory $\% 2 \mathrm{C}+$ reflected + in $+\mathrm{a}+$ cancer $+\mathrm{p}$ atient\%E2\%80\%99s+tweets.+BMC+Palliative+Care.

4. Chen $\mathrm{X}$, Lun $\mathrm{Y}$, Yan J, Hao T, Weng H. Discovering thematic change and evolution of utilizing social media for healthcare research. BMC Med Inform Decis Mak [Internet]. 2019 Apr [cited 2019 Mar 5];19(Suppl 2):50. Available from: https://bmcmedinformdecismak.biomedcentral.com/articles /10.1186/ s12911-019-0757-4. 
5. Taubert M, Watts G, Boland J, Radbruch L. Palliative social media. BMJ Support Palliat Care [Internet]. 2014 Mar [cited 2019 Mar 5];4(1):13-8. Available from: https://spcare.bmj.com/ content/ bmjspcare/4/1/13.full.pdf.

6. Mendes KDS, Silveira RCCP, Galvão CM. Revisão integrativa: método de pesquisa para a incorporação de evidências na saúde e na enfermagem. Texto \& contexto enferm [Internet]. 2008 out.-dez. [citado 5 mar 2019];17(4):758-64. Disponível em: http://www.scielo.br/pdf/tce/ v17n4/18.pdf.

7. Carrasco JM, Gómez-Baceiredo B, Navas A, Krawczyk M, García M, Centeno C. Social representation of palliative care in the Spanish printed media: A qualitative analysis. PLoS One [Internet]. 2019 Jan [cited 2019 Mar 11];14(1):e0211106. DOI: https://doi.org/10.1371/journal. pone.0211106.

8. Levy K, Grant PC, Depner RM, Tenzek KE, Pailler ME, Beaupin LK, Byrwa DJ. The Photographs of Meaning Program for Pediatric Palliative Caregivers: Feasibility of a Novel Meaning-Making Intervention. Am J Hosp Palliat Care [Internet]. 2019 Jan [cited 2019 Mar 11];36(7):557-63. DOI: https://doi.org/10.1177/1049909118824560.

9. Cleary JF, Maurer MA. Pain and policy studies group: two decades of working to address regulatory barriers to improve opioid availability and accessibility around the world. J Pain Symptom Manage [Internet]. 2018 Feb [cited 2019 Mar 11];55(2S):S121-S134. Available from: https://www.jpsmjournal. com/article/S0885-3924(17)30373-1/pdf.

10. Matthews M, Peters C, Lawson S. An exploratory investigation of hospice marketing: How are palliative care providers marketing their services? Health Mark Q [Internet]. 2017 Feb [cited 2019 Mar 11];34:4861. Available from: http://dx.doi.org/10.1080/07359683.2016.1275218.

11. Williams L, Gott M, Moeke-Maxwell T, Black S, Kothari S, Pearson S, Morgan T, Wharemate MR, Hansen W. Can digital stories go where palliative care research has never gone before? A descriptive qualitative study exploring the application of an emerging public health research method in an indigenous palliative care context. BMC Palliat Care [Internet]. 2017 [cited 2019 Mar 11];16:46. Available from: https:// bmcpalliatcare.biomedcentral.com/track/pdf/10.1186/s12904-017-0216-x.

12. Chan M, Fassbender K. Evaluating Public Engagement for a Consensus Development Conference. J Palliat Med [Internet]. 2018 Jan [cited 2019 Mar 11];21(S1):S20-S26. Available from: https://www. liebertpub.com/doi/pdfplus/10.1089/jpm.2017.0390.

13. Nwosu AC, Monnery D, Reid VL, Chapman L. Use of podcast technology to facilitate education, communication and dissemination in palliative care: the development of the AmiPal podcast. BMJ Support Palliat Care [Internet]. 2017 [cited 2019 Mar 11];7(2):212-17. DOI: https://doi.org/10.1136/ bmjspcare-2016-001140.

14. Guo Q, Cann B, McClement S, Thompson G, Chochinov HM. Keep in touch (KIT): perspectives on introducing internet-based communication and information technologies in palliative care. BMC Palliat care [Internet]. 2016 [cited 2019 Mar 11];15:66. DOI: https://doi.org/10.1186/s12904-016-0140-5.

15. Jamwal NR, Kumar SP. Maintaining the Social Flow of Evidence-Informed Palliative Care: Use and Misuse of YouTube. Indian J Palliat Care [Internet]. 2016 Jan-Mar [cited 2019 Mar 11]; 22(1):104-7. DOI: https://doi.org/10.4103/0973-1075.173945.

16. Hopewell-Kelly N, Baillie J, Sivell S, Harrop E, Bowyer A, Taylor S, Thomas K, Newman A, Prout H, Byrne A, Taubert M, Nelson A. Palliative care research centre's move into social media: constructing a framework for ethical research, a consensus paper. BM] Support Palliat Care [Internet]. 2016 Jan [cited 2019 Mar 11];9(2):219-24. Available from: https://spcare.bmj.com/content/bmjspcare/ early/2016/01/28/bmjspcare-2015-000889.full.pdf.

17. Nwosu AC, Debattista M, Rooney C, Mason S. Social media and palliative medicine: a retrospective 2-year analysis of global Twitter data to evaluate the use of technology to communicate about issues at the end of life. BMJ Support Palliat Care [Internet]. 2015 June [cited 2019 Mar 11];5(2):207-12. DOI: https://doi.org/10.1136/bmjspcare-2014-000701.

18. Comabella CC, Wanat M. Using social media in supportive and palliative care research. BMJ Support Palliat Care [Internet]. 2015 June [cited 2019 Mar 11];5(2):138-45. DOI: https://doi.org/10.1136/ bmjspcare-2014-000708.

19. Castells M, Medeiros CA. Redes de indignação e esperança: movimentos sociais na era da internet. Rio de Janeiro: Zahar, 2013. 271p. 
20. Zalewski K, Lindemann K, Halaska MJ, Zapardiel I, Laky R, Chereau E, Lindquist D, Polterauer S, Sukhin V, Dursun P. A Call for New Communication Channels for Gynecological Oncology Trainees: A Survey on Social Media Use and Educational Needs by the European Network of Young Gynecological Oncologists. Int J Gynecol Cancer [Internet]. 2017 Mar [cited 2019 Mar 11]; 27(3):620-26. DOI: https://doi. org/10.1097/IGC.0000000000000917.

21. Azevedo C, Pessalacia JDR, Mata LRF, Zoboli ELCP, Pereira MG. Interface entre apoio social, qualidade de vida e depressão em usuários elegíveis para cuidados paliativos. Rev. esc. enferm. USP [Internet]. 2017 [cited 2020 May 04];51:e03245. DOI: http://dx.doi.org/10.1590/s 1980-220x2016038003245.

22. Ituassu A, Lifschitz S, Capone L, Vaz MB, Mannheimer V. Compartilhamento de mídia e preferência eleitoral no Twitter: uma análise de opinião pública durante as eleições de 2014 no Brasil. Palabra Clave. 2018 [citado em 2020 maio 04];21(3):860-884. DOI: https://dx.doi.org/10.5294/ pacla.2018.21.3.9.

23. Schweiger S, Cress U. How Confidence in Prior Attitudes, Social Tag Popularity, and Source Credibility Shape Confirmation Bias Toward Antidepressants and Psychotherapy in a Representative German Sample: randomized controlled web-based study. J Med Internet Res [Internet]. 2019 Apr [cited 2019 Mar 11];21(4):e11081. DOI: https://doi.org/10.2196/11081.

24. Castells M. A sociedade em rede. São Paulo: Paz e Terra, 1999.

25. França T, Rabello ET, Magnago C. As mídias e as plataformas digitais no campo da Educação Permanente em Saúde: debates e propostas. Saúde debate [Internet]. 2019 Ago [citado em 2020 maio 04];43(spe1):106-15. DOI: https://doi.org/10.1590/0103-11042019s109.

26. Cawcutt KA, Erdahl LM, Englander MJ, Radford DM, Oxentenko AS, Girgis L, Migliore LL, Poorman JA, Silver JK. Use of a Coordinated Social Media Strategy to Improve Dissemination of Research and Collect Solutions Related to Workforce Gender Equity. J Women's Health (Larchmt) [Internet]. 2019 June [cited 2019 Mar 11];28(6):849-62. DOI: https://doi.org/10.1089/jwh.2018.7515. 